Research Article

\title{
An Interdisciplinary Pain Rehabilitation Program for Veterans with Chronic Pain: Description and Initial Evaluation of Outcomes
}

\author{
Nidhi S. Anamkath $\left(\mathbb{D},{ }^{1,2}\right.$ Sarah A. Palyo, ${ }^{1,2}$ Sara C. Jacobs, ${ }^{1,2}$ Alain Lartigue, \\ Kathryn Schopmeyer, ${ }^{1}$ and Irina A. Strigo $\mathbb{1}^{1,2}$ \\ ${ }^{1}$ Department of Veterans Affairs, San Francisco VA Healthcare System, 4150 Clement Street, San Francisco, CA, USA \\ ${ }^{2}$ University of California, San Francisco, CA, USA \\ Correspondence should be addressed to Irina A. Strigo; irina.strigo@ucsf.edu
}

Received 27 November 2017; Revised 13 March 2018; Accepted 18 March 2018; Published 17 April 2018

Academic Editor: Anna Maria Aloisi

Copyright (c) 2018 Nidhi S. Anamkath et al. This is an open access article distributed under the Creative Commons Attribution License, which permits unrestricted use, distribution, and reproduction in any medium, provided the original work is properly cited.

\begin{abstract}
Objective. Chronic pain conditions are prominent among Veterans. To leverage the biopsychosocial model of pain and comprehensively serve Veterans with chronic pain, the San Francisco Veterans Affairs Healthcare System has implemented the interdisciplinary pain rehabilitation program (IPRP). This study aims to (1) understand initial changes in treatment outcomes following IPRP, (2) investigate relationships between psychological factors and pain outcomes, and (3) explore whether changes in psychological factors predict changes in pain outcomes. Methods. A retrospective study evaluated relationships between clinical pain outcomes (pain intensity, pain disability, and opioid use) and psychological factors (depressive symptoms, catastrophizing, and "acceptable" level of pain) and changes in these outcomes following treatment. Multiple regression analysis explored whether changes in psychological variables significantly predicted changes in pain disability. Results. Catastrophizing and depressive symptoms were positively related to pain disability, while "acceptable" level of pain was idiosyncratically related to pain intensity. Pain disability and psychological variables showed significant changes in their expected directions. Regression analysis indicated that only changes in depressive symptoms significantly predicted changes in pain disability. Conclusion. Our results are consistent with evidence-based clinical practice guidelines for the management of chronic pain in Veterans. Further investigation of interdisciplinary treatment programs in Veterans is warranted.
\end{abstract}

\section{Introduction}

Chronic pain is a highly prevalent condition estimated to affect over $50 \%$ of Veterans who receive care through the Veterans Health Administration (VHA) [1,2]. In addition, an investigation by Nahin [3] found that Veterans not only have high rates of pain but also generally report more pain than non-Veterans and have increased rates of severe pain. There is a large body of evidence showing that pain is associated with a plethora of deleterious consequences, including affective distress, long-term opioid use, greater utilization of healthcare services, and significant financial distress for patients and society $[1,4,5]$. More concerning is that the incidence of persistent pain in Veterans seems to be growing and standalone treatments, such as medication, physical therapy, or Cognitive-Behavioral Therapy may not be adequate for all patients $[2,5,6]$. To this end, the VHA has implemented a National Pain Management Strategy calling for integrated treatment to specifically improve pain management for Veterans nationwide $[7,8]$.

Pain is a multifaceted experience affected by genetic and biological vulnerabilities, as well as by psychosocial factors [9]. Thus, treatments that use a biopsychosocial model in the treatment of chronic pain have been recommended and have demonstrated good clinical outcomes [9-12]. Specifically, integrated biopsychosocial treatments are designed to facilitate functional restoration by addressing not only physiological processes of pain but also the cognitive 
appraisals and emotional reactions which may exacerbate the pain experience $[13,14]$. One such psychological process known to affect the pain experience is pain catastrophizing, which is a multidimensional construct reflecting a collection of negative cognitions one has about experienced or anticipated pain [15]. Pain catastrophizing has shown to be a robust predictor of pain-related outcomes such as analgesic use and disability $[15,16]$. Likewise, depression is another important psychological process, which has a dramatic negative effect on the pain experience $[13,17,18]$. A review of the comorbidity between depression and pain by Bair et al. [19] indicates that those with depression experience more intense pain for longer periods of time. Additionally, experimental investigations repeatedly show that individuals with depression have increased emotional reactivity to experimentally induced pain compared to never depressed controls $[13,17]$. These results reinforce abnormal emotional processing in response to pain in those with depression, which therefore may exacerbate the pain experience for these individuals [20]. Of note, although often positively correlated, depression and catastrophizing have been shown to uniquely contribute to the pain experience, and thus, these two psychosocial facets should be examined separately [21-23]. Finally, one's perceived "acceptable" level of pain may also contribute to the pain experience. Clinically, "acceptable" level of pain is often assessed as a means of understanding patients' expectations for their pain care. Expectations regarding pain treatment and outcomes may drive motivations, adherence, and coping behaviors, thereby affecting overall treatment outcomes [24-26]. As such, it is crucial to understand how changes in one's expectation for what is an "acceptable level of pain" affect clinical pain outcomes.

The evidenced interplay between physiological, emotional, and cognitive aspects of pain warrants that these various components be addressed through treatment. Thus, the VHA has implemented interdisciplinary pain rehabilitation programs informed by the biopsychosocial model of pain to better address these different aspects of Veterans' pain experience [8]. Within the San Francisco Veterans Affairs Healthcare System (SFVAHCS), one such program, the Intensive Pain Rehabilitation Program (IPRP), was implemented in 2012 to address the increasing pain rehabilitation needs of Veterans. IPRP consists of several treatment components including the following: Acceptance and Commitment Therapy, Cogntive-Behavioral Therapy, physical therapy, pain education, and pharmacy counseling. One goal of the program has been to specifically address cognitive and emotional factors that may impact clinical pain outcomes such as pain intensity, pain-related disability, and current opioid medication use in Veterans with chronic, noncancer pain. Prior meta-analyses, which included studies mostly examining non-Veterans populations, have shown psychological interventions to be successful in reducing pain intensity, physical disability, and pain behaviors in individuals with chronic, musculoskeletal, noncancer pain $[27,28]$. Several factors, such as decreased cognitive distortions and increased psychological flexibility, have been identified as potential mechanisms by which these treatment changes occur $[29,30]$. However, there is a dearth of literature examining the relationship between cognitive and emotional psychological factors and clinical pain outcomes, particularly in Veterans receiving the highest, or "tertiary," level of integrated pain rehabilitation. Such a treatment may be best suited to address the complex nature of the pain experience, especially in the veteran population, which has been shown to report more pain than the non-Veterans population [3]. Thus, to expand upon the current literature conducted primarily in the non-Veterans population, further investigation of treatment outcomes specifically in Veterans who attend an integrated pain rehabilitation program, such as IPRP, is warranted.

The present observational study had three goals. The first was to examine the relationship between clinical pain outcomes (pain intensity, pain-related disability, and opioid medication use) and cognitive and emotional psychological factors (subjective "acceptable" pain level, pain catastrophizing, and depressive symptoms) in Veterans undergoing a 12-week intensive interdisciplinary treatment at both baseline and follow-up. The second goal was to examine whether there were statistically significant changes in clinical pain outcomes, as well as in cognitive and emotional variables following treatment. The third goal was to explore changes in which psychological factors best predicted changes in clinical pain outcomes. An evolving understanding of the relationships between these factors in a veteran sample undergoing such integrated rehabilitation may further inform treatment development, assessments, and protocols.

\section{Methods}

Study procedures were approved by the SFVAHCS and University of California San Francisco Institutional Review Boards. Patient data were gathered from the Intensive Pain Rehabilitation Program (IPRP), which is an intensive and interdisciplinary treatment program designed for patients receiving care through the SFVAHCS who suffer from functionally impairing chronic, noncancer pain conditions. Inclusion in the program requires a referral from a clinician within the Veterans Affairs Healthcare System and an IPRP team-based evaluation to determine an individual's fit for the program. The patients in this sample attended the program three half-days a week for twelve weeks and were provided with education and self-management skills to facilitate practice at home. Skills were designed to help patients meet functional goals. All patients received the following components as part of the program: physical therapy (PT), CBT, Acceptance and Commitment Therapy (ACT), pain education (PEd), and pharmacy counseling (PharmC). PT included instructions on gentle movements, novel movement strategies, and self-applied massage techniques designed to increase body awareness, recover ease of movement, and re-engage in daily physical activities without causing a significant increase in pain. CBT focused on introduction of skills such as activity pacing, activity scheduling, relaxation training, and cognitive restructuring. Distraction was de-emphasized to be consistent with ACT, 
which focuses on participants developing mindfulness skills, being present-focused, and engaging in valued activities. PEd provided participants with education about chronic pain, including neurophysiology and neuroplasticity concepts, as well as healthy lifestyle choices as they relate to living with chronic pain. PharmC included education about the balancing risk and benefits of pain medications through group classes and individualized counseling with optimization of pain medication regimens when appropriate. Of note, opioid use reduction was not an explicit treatment goal of IPRP; however, IPRP supported the reduction of opioid medication as a patient-initiated goal or if a provider identified safety concerns.

As part of their clinical care, patients completed pre- and posttreatment measures. Data for this study were retrospectively examined from the clinical data collected by the program. Of the 55 patients who enrolled in the 3-day/week IPRP program between December 2012 and May 2015, individuals were excluded from data analysis if (1) they dropped out, (2) they completed the program but had not completed posttreatment questionnaires, (3) more than 15\% of a questionnaire was missing either pre- or posttreatment (4) they were not Veterans, or (5) they were re-enrolled in the program (only the first enrollment was included in analyses to control for repetition effects). The final sample included 35 participants.

\subsection{Measures}

2.1.1. Demographic and Clinical Data. Patients self-reported clinical and demographic information both pre- and posttreatment. Information included age, sex, race, years of education completed, duration of pain, and identified pain sites. Additionally, average or "usual" pain intensity (during the past week) and "acceptable" pain level were reported using a numeric rating scale (0-10). Of note, "acceptable" level of pain refers to an anticipated pain score with which a patient would be comfortable rather than acceptance of their pain condition. Here, it is a measure of patients' expectations regarding treatment.

2.1.2. Pain Disability. The Pain Disability Questionnaire (PDQ) [31] is a 15-item measure of pain-related disability. Each item is rated from 0 to 10 with higher scores indicating greater pain-related disability. The measure is divided into two subscales. The first is the functional status component which reflects general functioning; the second is the psychosocial component. Scores on the PDQ are broken down to categorize pain disability as mild/moderate $(0-70)$, severe (71-100), or extreme (101-150) [32].

2.1.3. Pain Catastrophizing. The Pain Catastrophizing Scale (PCS) [15] is a 13-item self-report measure comprising three subscales used to assess an individual's negative cognitions (rumination, magnification, and hopelessness) about actual or anticipated pain. Items are rated using a 5-point Likert scale $(0=$ not at all and $4=$ all the time). The PCS is a widely used measure of pain cognitions amongst a variety of chronic pain populations and has been shown to have good internal consistency $[15,33]$.

2.1.4. Depression. The Patient Health Questionnaire (PHQ9) [34] is a nine-item self-report measure of depressive symptoms. Questions correspond to diagnostic criteria from the Diagnostic and Statistical Manual of Mental Disorders, 4th edition. Each item is rated from 0 to $3(0=$ not at all and $3=$ nearly every day). Scores are broken down into five categories: minimal depression (0-4); mild depression (5-9); moderate depression (10-14); moderately severe depression (15-19); and severe depression (20-27). The questionnaire has been shown to be reliable and has good sensitivity and specificity $[34,35]$.

2.1.5. Pharmacy Data. A pharmacist collected information on the number and type of medications that patients were taking specifically for their pain. Medication types included opioids, antidepressants, anticonvulsants, muscle relaxants, nonsteroidal anti-inflammatory drugs (NSAIDS), topical agents, and a category for other miscellaneous pain relievers. In addition, morphine daily equivalent doses (MEDD) were calculated for each patient as a measure of opioid medication use.

2.1.6. Treatment Satisfaction. Five questions from the Pain Outcomes Questionnaire [36] which reflect treatment satisfaction (TxSat) were asked posttreatment. Each question asks participants to rate their satisfaction of the care they received on a scale from 0 to $10(0=$ no satisfaction and $10=$ complete satisfaction). The questions ask patients to rate their satisfaction with the overall treatment, staff (personality and competence), and treatment schedule, as well as whether they would recommend the treatment. Rating of individual components of the program was not administered to this cohort but is being implemented currently.

2.2. Data Analysis. All statistical analyses were performed using IBM SPSS Statistics Version 24 (IBM, Chicago, IL). Parametric tests were used for all analyses. Demographic information and descriptive statistics for all outcome measures have been provided. Pretreatment correlations and posttreatment Pearson's correlations were investigated amongst variables (usual pain, acceptable pain, PDQ, PCS, PHQ9, MEDD, and TxSat). To assess differences in pre- and posttreatment scores for outcome variables, paired $t$-tests were performed. For all analyses, $p<0.05$ was considered significant.

Lastly, due to the interdisciplinary nature of the IPRP which aimed to improve cognitive and emotional symptoms associated with chronic pain, we wanted to explore changes in what emotional and cognitive symptoms best predicted changes in pain outcomes in our study. Hence, a multiple linear regression model was conducted for pre-post changes in pain-related disability as a pain outcome and acceptable level of pain, PHQ9, and PCS as psychosocial predictors. 
TABLE 1: Veteran sample characteristics $(n=35)$.

\begin{tabular}{|c|c|c|}
\hline & Pretreatment & Posttreatment \\
\hline \multicolumn{3}{|l|}{ Demographic } \\
\hline Mean age in years (SD) & $56.2(7.9)$ & - \\
\hline Male sex, $n(\%)$ & $25(71 \%)$ & - \\
\hline Caucasian race, $n(\%)$ & $22(62.9 \%)$ & - \\
\hline Mean pain duration (SD), $n=33^{\#}$ & $18.70(13.20)$ & \\
\hline Mean number of pain sites (SD) & $7.89(4.40)$ & $6.94(4.26)$ \\
\hline \multicolumn{3}{|l|}{ Reported pain sites, $f(\%)$} \\
\hline Leg & $26(74.29 \%)$ & $26(74.29 \%)$ \\
\hline Low back & $32(91.43 \%)$ & $30(85.71 \%)$ \\
\hline Mid-back & $22(62.86 \%)$ & $18(51.43 \%)$ \\
\hline Upper back & $17(48.57 \%)$ & $12(34.29 \%)$ \\
\hline Head & $12(34.29 \%)$ & $9(25.71 \%)$ \\
\hline Neck & $25(71.43 \%)$ & $20(57.14 \%)$ \\
\hline Shoulder & $19(54.29 \%)$ & $20(57.14 \%)$ \\
\hline Buttocks & $19(54.29 \%)$ & $12(34.29 \%)$ \\
\hline Foot & $15(42.86 \%)$ & $16(45.71 \%)$ \\
\hline Jaw & $10(28.57 \%)$ & $7(20.00 \%)$ \\
\hline Chest & $6(17.14 \%)$ & $3(8.57 \%)$ \\
\hline Abdomen & $7(20.00 \%)$ & $4(11.43 \%)$ \\
\hline Arm/hand & $17(48.57 \%)$ & $13(37.14 \%)$ \\
\hline Fingers & $14(40.00 \%)$ & $10(28.57 \%)$ \\
\hline Toes & $12(34.29 \%)$ & $7(20.00 \%)$ \\
\hline Face & $4(11.43 \%)$ & $2(5.71 \%)$ \\
\hline Genitals & $6(17.14 \%)$ & $5(14.29 \%)$ \\
\hline Others & $8(22.86 \%)$ & $8(22.86 \%)$ \\
\hline \multicolumn{3}{|l|}{ Medications, $f(\%)$} \\
\hline Opioids & $26(74.29 \%)$ & $25(71.43 \%)$ \\
\hline Antidepressants & $16(45.71 \%)$ & $18(51.43 \%)$ \\
\hline Anticonvulsants & $17(48.57 \%)$ & $17(48.57 \%)$ \\
\hline Muscle relaxants & $14(40.00 \%)$ & $16(45.71 \%)$ \\
\hline NSAID & $13(37.14 \%)$ & $15(42.86 \%)$ \\
\hline Topical agents & $20(57.14 \%)$ & $22(62.86 \%)$ \\
\hline Others & $8(22.86 \%)$ & $8(22.86 \%)$ \\
\hline \multicolumn{3}{|l|}{ Clinical pain outcomes, mean (SD) } \\
\hline Usual pain ${ }^{\&}$ & $6.40(1.94)$ & $5.76(1.69), n=34^{\#}$ \\
\hline PDQ & $103.14(23.01)$ & $87.77(24.40)$ \\
\hline MEDD & $69.68(100.88), n=34^{\#}$ & $62.32(91.90), n=34^{\#}$ \\
\hline \multicolumn{3}{|l|}{ Psychological outcomes, mean (SD) } \\
\hline PHQ9 & $14.29(6.23), n=34^{\#}$ & $9.89(5.50)$ \\
\hline PCS & $23.78(12.21), n=32^{\#}$ & $13.85(8.46), n=33^{\#}$ \\
\hline Acceptable pain ${ }^{\&}$ & $2.63(1.50), n=32^{\#}$ & $3.76(1.50), n=34^{\#}$ \\
\hline \multicolumn{3}{|l|}{ Others } \\
\hline Treatment satisfaction & - & $46.06(5.99)$ \\
\hline
\end{tabular}

$\mathrm{SD}=$ standard deviation; NSAID = nonsteroidal anti-inflammatory drugs; PHQ9= Patient Health Questionnaire; PCS= Pain Catastrophizing Scale; $\mathrm{PDQ}=$ Pain Disability Questionnaire; MEDD = morphine equivalent daily dose; ${ }^{8}$ numeric rating scale from 0 (no pain) to 10 (worst pain imaginable). ${ }^{*}$ The number of patients is lower than the total number of patients $(n=35)$.

Associations among individual demographic factors (age and sex) and outcomes were tested before running each regression to identify potential covariates. These predictors were chosen based on the components of the program that are meant to target cognitive and emotional symptoms of chronic pain.

\section{Results}

3.1. Veteran Sample Characteristics. Individuals in this sample were primarily male (71\%) and Caucasian $(62.9 \%)$. The mean age was 56.2 years $(\mathrm{SD}=7.9)$. Means and standard deviations for pre- and posttreatment variables are presented in Table 1. As can be seen in Table 1, on average, patients' self-reported scores at baseline (or pretreatment self-reported scores) indicated moderate depression and extreme disability. Average pain duration (in years) and number of pain sites reported were $18.7(\mathrm{SD}=13.2)$ and 7.9 $(\mathrm{SD}=4.4)$, respectively. Table 1 also depicts sample characteristics for medication use, which showed that majority of these patients were receiving opioids.

3.2. Bivariate Associations. Pre- and posttreatment bivariate correlations are depicted in Tables 2 and 3, respectively. Usual pain was significantly correlated with the acceptable 
TABLE 2: Pretreatment correlations.

\begin{tabular}{lcccccc}
\hline Variable & 1 & 2 & 3 & 4 & 5 & 6 \\
\hline (1) Usual pain & - & $0.39^{*}$ & 0.20 & 0.08 & $0.39^{*}$ & -0.08 \\
(2) Acceptable pain & & - & 0.03 & -0.07 & 0.26 & -0.08 \\
(3) PHQ & & & - & $0.54^{* *}$ & $0.64^{* *}$ & 0.15 \\
(4) PCS & & & & - & $0.51^{* *}$ & 0.10 \\
(5) PDQ & & & & & - & 0.25 \\
(6) MEDD & & & & & & -
\end{tabular}

PHQ9 = Patient Health Questionnaire; PCS = Pain Catastrophizing Scale; $\mathrm{PDQ}=$ Pain Disability Questionnaire; $\mathrm{MEDD}=$ morphine equivalent daily dose; ${ }^{\&}$ numeric rating scale from 0 (no pain) to 10 (worst pain imaginable); *correlation is significant at the 0.05 level $\left(2\right.$-tailed); ${ }^{* *}$ correlation is significant at the 0.01 level (2-tailed).

TABle 3: Posttreatment correlations.

\begin{tabular}{lcccccc}
\hline Variable & 1 & 2 & 3 & 4 & 5 & 6 \\
\hline (1) Usual pain $^{8}$ & - & 0.34 & 0.21 & 0.29 & $0.36^{*}$ & -0.17 \\
(2) Acceptable pain & & - & -0.18 & -0.14 & 0.01 & 0.05 \\
(3) PHQ & & & - & $0.45^{* *}$ & $0.50^{* *}$ & 0.31 \\
(4) PCS & & & & - & 0.34 & -0.08 \\
(5) PDQ & & & & & - & 0.20 \\
(6) MEDD & & & & & & - \\
\hline
\end{tabular}

PHQ9 = Patient Health Questionnaire; PCS = Pain Catastrophizing Scale; $\mathrm{PDQ}=$ Pain Disability Questionnaire; $\mathrm{MEDD}=$ morphine equivalent daily dose; ${ }^{*}$ numeric rating scale from 0 (no pain) to 10 (worst pain imaginable); ${ }^{*}$ correlation is significant at the 0.05 level (2-tailed); ${ }^{* *}$ correlation is significant at the 0.01 level (2-tailed).

TABle 4: Examination of changes in pre- and posttreatment measures.

\begin{tabular}{|c|c|c|c|}
\hline & $t$ & df & Significance (2-tailed) \\
\hline Usual pain ${ }^{\&}$ & 1.77 & 33 & 0.09 \\
\hline Acceptable pain ${ }^{\&}$ & $-4.87^{*}$ & 31 & 0.00 \\
\hline PHQ9 & $4.47^{*}$ & 33 & 0.00 \\
\hline PCS & $4.75^{*}$ & 29 & 0.00 \\
\hline PDQ & $4.38^{*}$ & 34 & 0.00 \\
\hline MEDD & 1.88 & 33 & 0.07 \\
\hline
\end{tabular}

PHQ9 = Patient Health Questionnaire; PCS = Pain Catastrophizing Scale; $\mathrm{PDQ}=$ Pain Disability Questionnaire; $\mathrm{MEDD}=$ morphine equivalent daily dose; ${ }^{8}$ numeric rating scale from 0 (no pain) to 10 (worst pain imaginable); ${ }^{*}$ correlation is significant at the 0.05 level (2-tailed).

level of pain only at baseline $(r=0.39, p<0.05)$ but not with PCS or PHQ at neither pre- nor posttreatment. PDQ was significantly correlated with PHQ $(r=0.64, p<0.01)$ and PCS $(r=0.51, p<0.01)$ but was not significantly related to the acceptable level pain. Posttreatment correlations between PDQ and PCS remained positive, but only the relationship to PDQ and PHQ remained significant $(r=0.50, p<0.01)$. MEDD did not significantly relate to any of the psychological outcomes both pre- and posttreatment in our sample.

3.3. Comparison of Pre-and Posttreatment Outcomes. Paired $t$-tests for pre- and posttreatment values from completed self-report measures are presented in Table 4. Results indicate significant decreases in all variable scores except for "acceptable pain levels," which significantly increased, as
TABLE 5: Linear regression analysis predicting pre-post PDQ.

\begin{tabular}{|c|c|c|c|}
\hline & Coefficients & $t$ & Significance \\
\hline$\triangle \mathrm{PHQ9}$ & 0.45 & 2.90 & 0.00 \\
\hline$\Delta$ Acceptable pain ${ }^{\&}$ & -0.03 & -0.21 & 0.84 \\
\hline$\triangle \mathrm{PCS}$ & 0.18 & 1.11 & 0.28 \\
\hline
\end{tabular}

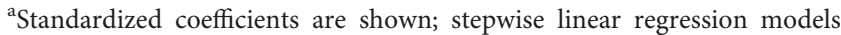
with changes in pain disability (PDQ) as dependent variables and three prepost predicting factors: depression (PHQ9), acceptable level of pain, and pain catastrophizing (PCS); $\mathrm{PHQ} 9=$ Patient Health Questionnaire; $\mathrm{PCS}=$ Pain Catastrophizing Scale; $\mathrm{PDQ}=$ Pain Disability Questionnaire;

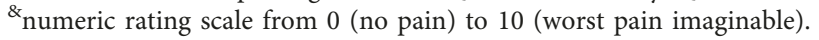

expected. Conversely, no significant changes were observed for pain intensity (usual pain) or for daily morphine equivalent dose (although tendencies were noted).

3.4. Exploratory Regression Analyses. The results of exploratory stepwise linear regression analysis with changes in pain disability (PDQ) as a dependent variable and changes in three predicting factors (depression, acceptable level of pain, and catastrophizing) are shown in Table 5. Improved pain disability scores (PDQ) were significantly predicted by improvements in depression scores, whereby changes in PHQ9 explained most of the changes in pain-related disability; adding acceptable levels of pain and/or catastrophizing did not improve the model.

\section{Discussion}

The present study retrospectively examines a sample of Veterans enrolled in interdisciplinary pain rehabilitation with the goal of exploring (1) the relationships between cognitive and emotional psychological variables and clinical pain outcomes, (2) changes in these psychological variables and outcomes following interdisciplinary treatment, and (3) whether changes in psychological variables predict changes in clinical pain outcomes. Expanding upon the existing literature, which has primarily evaluated interdisciplinary pain rehabilitation in the non-Veterans population, our findings indicate distinct relationships between pain-related clinical outcomes and the assessed psychological processes. Additionally, we found that the 12-week interdisciplinary pain rehabilitation program shows promise in improving pain-related psychological factors and pain-related disability in a mixed sample of extremely disabled, moderately depressed Veterans with severe chronic pain in multiple body sites. Lowering depressive symptoms may predict improvements in disability, and given the limited nonpharmacological options available to Veterans with such disability, these promising findings merit further examination.

We found several associations between clinical pain outcomes (i.e., self-reported pain intensity, pain-related disability, and opioid medication use) and the evaluated psychological measures, suggesting that pain-related clinical outcomes may be differentially influenced by the underlying cognitions and emotion. Specifically, pain intensity was significantly and positively associated with subjective acceptable pain level but not with pain catastrophizing or 
depressive symptom severity at both pre- and posttreatment. In contrast, pretreatment pain-related disability was positively related to depressive symptoms severity and pain catastrophizing but not with subjective acceptable level of pain. Such idiosyncratic relationships between the painrelated clinical outcomes and psychological features may have important implications for treatment planning based on a patient's specific goals. As an example, IPRP may choose to focus on functional restoration for a patient and use behavioral interventions (i.e., CBT and/or ACT) to target depressive symptom severity and pain catastrophizing, as these psychological processes were distinctly related to painrelated disability and therefore have the potential to impact pain-related debilitation if reduced. Targeting underlying processes that may affect the pain experience has indeed become a topic of interest in the literature [37], and further consideration of these distinctive relationships between pain outcomes and psychological processes has the potential to optimize treatment recommendations.

A comparison of pre- and posttreatment scores demonstrates that the administered intensive and interdisciplinary treatment significantly lessened negative cognitions and emotions associated with chronic pain, as well as subjects' perceived disability level, but had less effect on self-reported pain intensity and opioid use. Regarding the lack of improvement in pain intensity, this suggests that decreases in disability were not necessarily a function of decreases in pain intensity and is consistent with the extant literature and with the VHA National Pain Strategy [8], which calls for improvements in both physical and psychosocial functioning. Although decreases in pain intensity following interdisciplinary rehabilitation often occur, such reductions may not be necessary for improvements in functioning $[38,39]$. This directly supports the foundational theory of the Acceptance and Commitment Therapy (ACT) treatment model, which hypothesizes that one's orientation to a distressing experience can be altered without an alteration in the distressing experience itself $[29,40]$. Also, in line with the ACT model, decreases of negative psychological states, for example, depression and pain catastrophizing, may facilitate increases in behavioral flexibility. Increases in behavioral flexibility allow patients to re-engage in life activities reflecting personal values and thus decrease disability. Although behavioral flexibility was not measured in this initial sample of Veterans undergoing IPRP, it is plausible that our integrative treatment that improved depression and pain catastrophizing may have decreased disability by giving patients the option to engage in more value-based behaviors which they may have been avoiding previously [41, 42]. The significant impact that depression and catastrophizing may have on decreasing disability further supports the idea that decreases in pain intensity may not be needed for functional improvement.

Likewise, we found that opioid medication use did not show significant decrease although reduction tendencies were noted. Given the current state of the opioid epidemic $[43,44]$ and its relevance to the veteran population in particular [45-47], this observation warrants particular attention. Given that opioid dose reduction was not an explicit treatment goal of IPRP, lack of significant decrease in opioid medication use is not surprising. While there is evidence that similar interdisciplinary programs may be effective in reducing opioid intake, these programs often focus on opioid use reduction as part of their treatment or mandate cessation of opioids altogether [48, 49]. However, our findings are consistent with the existing literature [50] suggesting that decreases in opioid use may not be related to decreases in pain-related disability and/or improvements in psychological outcomes. This finding is promising as many patients who refuse to reduce opioid intake for fear of worsening of their pain symptoms may still be able to make functional gains. If such functional gains are made, patients may be more willing to initiate changes in their opioid use with the help of their treatment providers. Importantly, future follow-up investigations should examine whether participants require more time after treatment has completed to solidify newly acquired functional gains that would support greater reductions in opioids use.

Finally, exploratory regression analyses indicated that only changes in depressive symptoms severity significantly predicted changes in pain-related disability in the current sample. While other studies also found that changes in catastrophizing may also be predictive of improvements in pain disability, which we did not observe, our results lend further support to the strong relationship between depression and pain-related disability which has been noted in the existing literature [21, 51-53]. In line with the present finding, investigations have repeatedly found that depressive symptoms in patients with chronic pain are associated with increased levels of disability $[19,54]$. Additionally, a longitudinal investigation of a non-Veterans sample by Scott et al. [53] found that reductions in depression significantly predicted both decreases in disability days and decreased likelihood of severe disability. Contrary to our findings, Scott et al. [53] found that levels of pain catastrophizing also predicted decreased levels of high disability and disability days, although the effect size was moderate compared to the effects of depression in their study. Thus, focusing on lowering depressive symptoms through value-based actions, cognitive restructuring, and other psychological techniques may be critical in increasing functional improvements in veteran population. Yet another study showed that that catastrophizing had a larger role in predicting disability levels than depression [21]. Although the findings regarding whether depression or catastrophizing is more important for pain-related disability are mixed, the literature is consistent in indicating that both psychological factors play an important role. Thus, future investigations of catastrophizing in a larger sample of Veterans in interdisciplinary treatment may indeed predict changes in disability.

Several limitations of the present study should be noted. Firstly, the current sample was restricted to those patients who had minimal missing data $(<10 \%)$, which limited the research and associated conclusions in the following ways: (a) possible introduction of selection bias, (b) limited sample size, and (c) lack of posttreatment follow-up data. Such limitations indeed impact the generalizability of our findings, and further research with a larger and more inclusive 
sample is needed. Thus, our initial findings should be interpreted with caution, particularly regarding the nonsignificant reduction in average reported pain intensity. We are currently collecting data in a larger sample of Veterans and at multiple assessment time points. Additionally, a future examination with a larger and less restrictive sample would also allow for a more fine-grained analysis of the outcome variables and identification of potential mechanisms of change. Furthermore, all data collected were selfreported, and results are subject to possible over- and underreporting. Future examinations should include additional objective measures of functioning, such as physical therapy outcomes. Despite these limitations, these initial results are promising and clearly demonstrate the need for further evaluations in our veteran population. Specifically, due to apparent lack of comparative efficacy trials for nonpharmacological pain treatment options available to our Veterans, the investigation of the efficacy of such a program via a randomized controlled trial is desperately needed.

\section{Conclusion}

Chronic pain is a major concern among Veterans, leading to immense suffering and disability. Interdisciplinary treatment of chronic pain has been recommended by the VHA, and appraisal of such a nonpharmacological treatment in this specific population is needed. Preliminary evaluation of such a treatment program shows intensive and interdisciplinary pain rehabilitation to be a promising treatment for Veterans with chronic pain. Patients overall exhibited positive gains in cognitions and emotions related to their pain experience, as well as improved functioning. These improvements among this sample are promising and are a call to action to conduct efficacy trials going forward.

\section{Disclosure}

The views expressed in this article are those of the authors and do not necessarily represent the position or policy of the Department of Veterans Affairs.

\section{Conflicts of Interest}

The authors declare that there are no conflicts of interest.

\section{Acknowledgments}

This work was supported in part by I01-CX-000816 (IAS) from the United States Department of Veterans Affairs Clinical Sciences Research and Development Service. The authors thank the University of California San Francisco Open Access Publishing Fund for the support.

\section{References}

[1] R. D. Kerns, E. J. Philip, A. W. Lee, and P. H. Rosenberger, "Implementation of the veterans health administration national pain management strategy," Translational Behavioral Medicine, vol. 1, no. 4, pp. 635-643, 2011.
[2] J. L. Goulet, R. D. Kerns, M. Bair et al., “The musculoskeletal diagnosis cohort: examining pain and pain care among veterans," Pain, vol. 157, no. 8, pp. 1696-1703, 2016.

[3] R. L. Nahin, "Severe pain in veterans: the effect of age and sex, and comparisons with the general population," Journal of Pain, vol. 18, no. 3, pp. 247-254, 2017.

[4] S. H. Snook and B. S. Webster, "The cost of disability," Clinical Orthopaedics and Related Research, vol. 221, pp. 77-84, 1987.

[5] K. Seal, W. Becker, J. Tighe, Y. Li, and T. Rife, "Managing chronic pain in primary care: it really does take a village," Journal of General Internal Medicine, vol. 32, no. 8, pp. 931934, 2017.

[6] H. Flor, T. Fydrich, and D. C. Turk, "Efficacy of multidisciplinary pain treatment centers: a meta-analytic review," Pain, vol. 49, no. 2, pp. 221-230, 1992.

[7] Interagency Pain Research Coordinating Committee, $\mathrm{Na}$ tional Pain Strategy: A Comprehensive Population HealthLevel Strategy for Pain, Department of Health and Human Services, Washington, DC, USA, 2015.

[8] Department of Veterans Affairs, Pain Management (VHA Directive 2009-053), Veterans Health Administration, Department of Veterans Affairs, Washington, DC, USA, 2009.

[9] R. J. Gatchel, Y. B. Peng, M. L. Peters, P. N. Fuchs, and D. C. Turk, "The biopsychosocial approach to chronic pain: scientific advances and future directions," Psychological Bulletin, vol. 133, no. 4, p. 581, 2007.

[10] R. J. Gatchel, D. D. McGeary, C. A. McGeary, and B. Lippe, "Interdisciplinary chronic pain management: past, present, and future," American Psychologist, vol. 69, no. 2, p. 119, 2014.

[11] R. J. Gatchel and A. Okifuji, "Evidence-based scientific data documenting the treatment and cost-effectiveness of comprehensive pain programs for chronic nonmalignant pain," Journal of Pain, vol. 7, no. 11, pp. 779-793, 2006.

[12] S. H. Sanders, R. N. Harden, and P. J. Vicente, "Evidencebased clinical practice guidelines for interdisciplinary rehabilitation of chronic nonmalignant pain syndrome patients," Pain Practice, vol. 5, no. 4, pp. 303-315, 2005.

[13] I. A. Strigo, A. N. Simmons, and S. C. Matthews, "Increased affective bias revealed using experimental graded heat stimuli in young depressed adults: evidence of "emotional allodynia”," Psychosomatic Medicine, vol. 70, no. 3, p. 338, 2008.

[14] C. Villemure and C. M. Bushnell, "Cognitive modulation of pain: how do attention and emotion influence pain processing?," Pain, vol. 95, no. 3, pp. 195-199, 2002.

[15] M. J. Sullivan, The Pain Catastrophizing Scale: User Manual, McGill University, Montreal, Canada, 2009.

[16] M. J. Sullivan, M. E. Lynch, A. J. Clark, T. Mankovsky, and J. Sawynok, "Catastrophizing and treatment outcome: differential impact on response to placebo and active treatment outcome," Contemporary Hypnosis, vol. 25, no. 3-4, pp. 129-140, 2008.

[17] A. Ushinsky, L. E. Reinhardt, A. N. Simmons, and I. A. Strigo, "Further evidence of emotional allodynia in unmedicated young adults with major depressive disorder," PLoS One, vol. 8, no. 11, article e80507, 2013.

[18] K. J. Bär, S. Brehm, M. K. Boettger, S. Boettger, G. Wagner, and H. Sauer, "Pain perception in major depression depends on pain modality," Pain, vol. 117, no. 1-2, pp. 97-103, 2005.

[19] M. J. Bair, R. L. Robinson, W. Katon, and K. Kroenke, "Depression and pain comorbidity: a literature review," Archives of Internal Medicine, vol. 163, no. 20, pp. 2433-2445, 2003. 
[20] M. L. Loggia, J. S. Mogil, and M. C. Bushnell, "Experimentally induced mood changes preferentially affect pain unpleasantness," Journal of Pain, vol. 9, no. 9, pp. 784-791, 2008.

[21] B. A. Arnow, C. M. Blasey, M. J. Constantino et al., "Catastrophizing, depression and pain-related disability," General Hospital Psychiatry, vol. 33, no. 2, pp. 150-156, 2011.

[22] R. R. Edwards, R. H. Dworkin, M. D. Sullivan, D. C. Turk, and A. D. Wasan, "The role of psychosocial processes in the development and maintenance of chronic pain," Journal of Pain, vol. 17, no. 9, pp. T70-T92, 2016.

[23] R. R. Edwards, C. Cahalan, G. Mensing, M. Smith, and J. A. Haythornthwaite, "Pain, catastrophizing, and depression in the rheumatic diseases," Nature Reviews Rheumatology, vol. 7, no. 4, pp. 216-224, 2011.

[24] S. J. Linton and W. S. Shaw, "Impact of psychological factors in the experience of pain," Physical Therapy, vol. 91, no. 5, pp. 700-711, 2011.

[25] C. J. Main, N. Foster, and R. Buchbinder, "How important are back pain beliefs and expectations for satisfactory recovery from back pain?," Best Practice \& Research Clinical Rheumatology, vol. 24, no. 2, pp. 205-217, 2010.

[26] J. E. Bialosky, M. D. Bishop, and J. A. Cleland, "Individual expectation: an overlooked, but pertinent, factor in the treatment of individuals experiencing musculoskeletal pain," Physical Therapy, vol. 90, no. 9, pp. 1345-1355, 2016.

[27] S. Morley, C. Eccleston, and A. Williams, "Systematic review and meta-analysis of randomized controlled trials of cognitive behaviour therapy and behaviour therapy for chronic pain in adults, excluding headache," Pain, vol. 80, no. 1, pp. 1-3, 1999.

[28] B. M. Hoffman, R. K. Papas, D. K. Chatkoff, and R. D. Kerns, "Meta-analysis of psychological interventions for chronic low back pain," Health Psychology, vol. 26, no. 1, p. 1, 2007.

[29] L. Dindo, J. R. Van Liew, and J. J. Arch, "Acceptance and commitment therapy: a transdiagnostic behavioral intervention for mental health and medical conditions," Neurotherapeutics, vol. 14, no. 3, pp. 546-553, 2017.

[30] K. E. Vowles, L. M. McCracken, and J. Z. O’Brien, “Acceptance and values-based action in chronic pain: a three-year follow-up analysis of treatment effectiveness and process," Behaviour Research and Therapy, vol. 49, no. 11, pp. 748-755, 2011.

[31] C. Anagnostis, R. J. Gatchel, and T. G. Mayer, "The pain disability questionnaire: a new psychometrically sound measure for chronic musculoskeletal disorders," Spine, vol. 29, no. 20, pp. 2290-2302, 2004.

[32] R. J. Gatchel, T. G. Mayer, and B. R. Theodore, "The pain disability questionnaire: relationship to one-year functional and psychosocial rehabilitation outcomes," Journal of Occupational Rehabilitation, vol. 16, no. 1, pp. 72-91, 2006.

[33] A. Osman, F. X. Barrios, P. M. Gutierrez, B. A. Kopper, T. Merrifield, and L. Grittmann, "The pain catastrophizing scale: further psychometric evaluation with adult samples," Journal of Behavioral Medicine, vol. 23, no. 4, pp. 351-365, 2000.

[34] K. Kroenke, R. L. Spitzer, and J. B. Williams, "The PHQ-9: validity of a brief depression severity measure," Journal of General Internal Medicine, vol. 16, pp. 606-613, 2001.

[35] A. Martin, W. Rief, A. Klaiberg, and E. Braehler, "Validity of the brief patient health questionnaire mood scale (PHQ-9) in the general population," General Hospital Psychiatry, vol. 28, no. 1, pp. 71-77, 2006.

[36] M. E. Clark, R. J. Gironda, and R. W. Young, "Development and validation of the pain outcomes questionnaire-VA,"
Journal of Rehabilitation Research and Development, vol. 40, no. 5 , p. 381, 2003.

[37] K. E. Weiss, A. Hahn, D. P. Wallace, B. Biggs, B. K. Bruce, and T. E. Harrison, "Acceptance of pain: associations with depression, catastrophizing, and functional disability among children and adolescents in an interdisciplinary chronic pain rehabilitation program," Journal of Pediatric Psychology, vol. 38, no. 7, pp. 756-765, 2013.

[38] K. E. Vowles, K. Witkiewitz, J. Levell, G. Sowden, and J. Ashworth, "Are reductions in pain intensity and painrelated distress necessary? An analysis of within-treatment change trajectories in relation to improved functioning following interdisciplinary acceptance and commitment therapy for adults with chronic pain," Journal of Consulting and Clinical Psychology, vol. 85, no. 2, p. 87, 2017.

[39] J. C. Ballantyne and M. D. Sullivan, "Intensity of chronic pain-the wrong metric?," New England Journal of Medicine, vol. 373, no. 22, pp. 2098-2099, 2015.

[40] J. D. Herbert, "Acceptance and Commitment Therapy: An Experiential Approach to Behavior Change," S. C. Hayes, K. D. Strosahl, and K. G. Wilson, Eds., Guilford Press, New York, NY, USA, 1999.

[41] R. K. Wicksell and K. E. Vowles, "The role and function of acceptance and commitment therapy and behavioral flexibility in pain management," Pain Management, vol. 5, no. 5, pp. 319-322, 2015.

[42] M. D. Sullivan and K. E. Vowles, "Patient action: as means and end for chronic pain care," Pain, vol. 158, no. 8, pp. 14051407, 2017.

[43] L. Laxmaiah, S. Helm, B. Fellows et al., "Opioid epidemic in the United States," Pain Physician, vol. 15, pp. 2150-1149, 2012.

[44] L. S. Nelson, D. N. Juurlink, and J. Perrone, "Addressing the opioid epidemic," JAMA, vol. 314, no. 14, pp. 1453-1454, 2015.

[45] J. L. Clarke, A. Skoufalos, and R. Scranton, “The American opioid epidemic: population health implications and potential solutions. Report from the National Stakeholder Panel," Population Health Management, vol. 19, no. S1, pp. S-1-S-10, 2016.

[46] A. S. Bohnert, M. A. Ilgen, J. A. Trafton et al., "Trends and regional variation in opioid overdose mortality among Veterans Health Administration patients, fiscal year 2001 to 2009," Clinical Journal of Pain, vol. 30, no. 7, pp. 605-612, 2014.

[47] K. H. Seal, Y. Shi, G. Cohen et al., "Association of mental health disorders with prescription opioids and high-risk opioid use in US veterans of Iraq and Afghanistan," JAMA, vol. 307, no. 9, pp. 940-947, 2012.

[48] J. W. Frank, T. I. Lovejoy, W. C. Becker et al., "Patient outcomes in dose reduction or discontinuation of long-term opioid therapy: a systematic review," Annals of Internal Medicine, vol. 167, no. 3, pp. 181-191, 2017.

[49] J. L. Murphy, K. M. Phillips, and S. Rafie, "Sex differences between Veterans participating in interdisciplinary chronic pain rehabilitation," Journal of Rehabilitation Research \& Development, vol. 53, no. 1, pp. 83-94, 2016.

[50] J. E. MacLaren, R. T. Gross, J. A. Sperry, and J. T. Boggess, "Impact of opioid use on outcomes of functional restoration," Clinical Journal of Pain, vol. 22, no. 4, pp. 392-398, 2006.

[51] M. Ericsson, W. S. C. Poston, J. Linder, J. E. Taylor, C. K. Haddock, and J. P. Foreyt, "Depression predicts disability in long-term chronic pain patients," Disability and Rehabilitation, vol. 24, no. 6, pp. 334-340, 2002. 
[52] M. N. Raftery, K. Sarma, A. W. Murphy, D. De la Harpe, C. Normand, and B. E. McGuire, "Chronic pain in the Republic of Ireland-community prevalence, psychosocial profile and predictors of pain-related disability: results from the Prevalence, Impact and Cost of Chronic Pain (PRIME) study, part 1," Pain, vol. 152, no. 5, pp. 1096-1103, 2011.

[53] E. L. Scott, K. Kroenke, J. Wu, and Z. Yu, "Beneficial effects of improvement in depression, pain catastrophizing, and anxiety on pain outcomes: a 12-month longitudinal analysis," Journal of Pain, vol. 17, no. 2, pp. 215-222, 2016.

[54] C. E. Dionne, T. D. Koepsell, M. Von Korff, R. A. Deyo, W. E. Barlow, and H. Checkoway, "Predicting long-term functional limitations among back pain patients in primary care settings," Journal of Clinical Epidemiology, vol. 50, no. 1, pp. 31-43, 1997. 


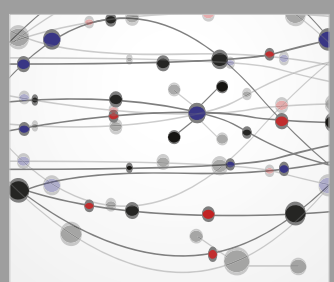

The Scientific World Journal
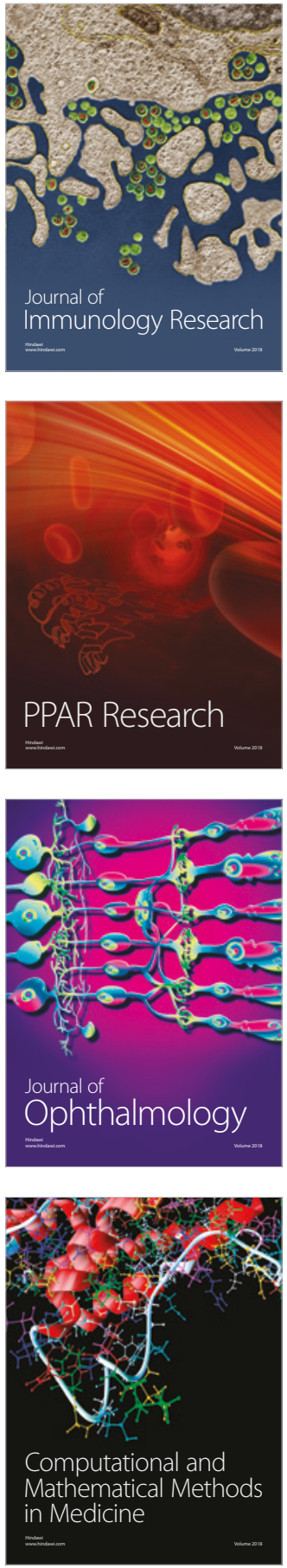

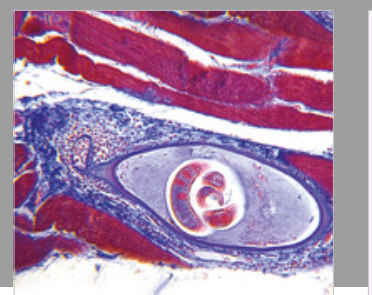

Gastroenterology Research and Practice

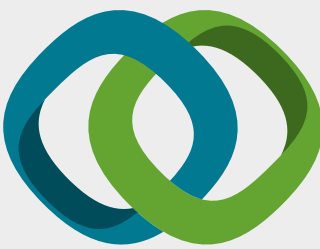

\section{Hindawi}

Submit your manuscripts at

www.hindawi.com
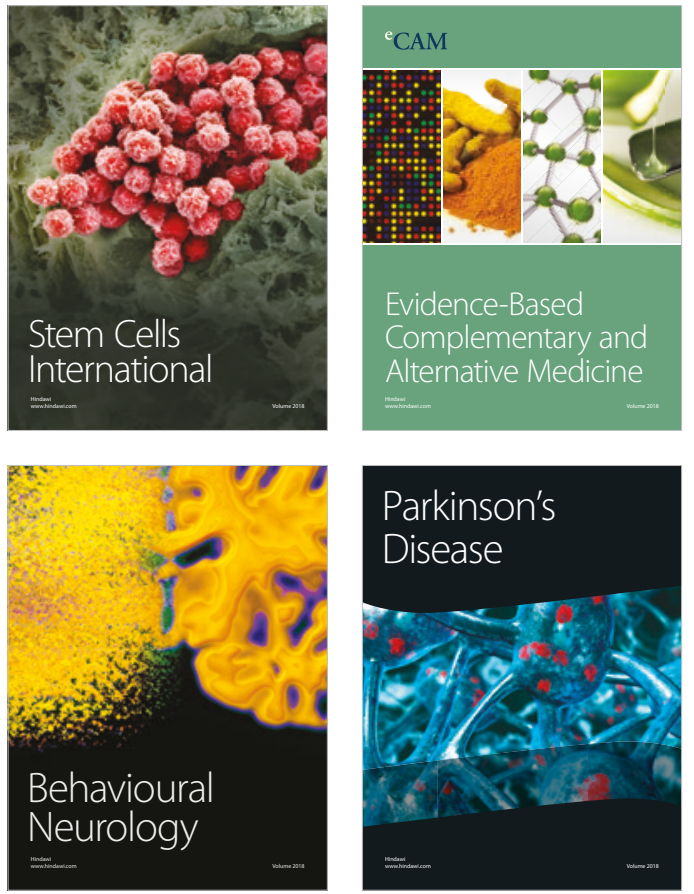

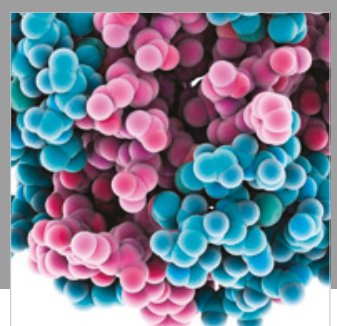

ournal of

Diabetes Research

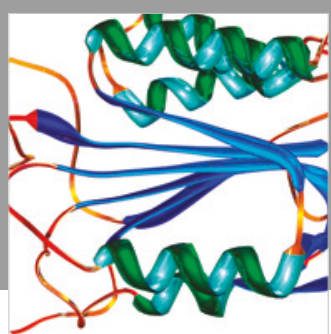

Disease Markers
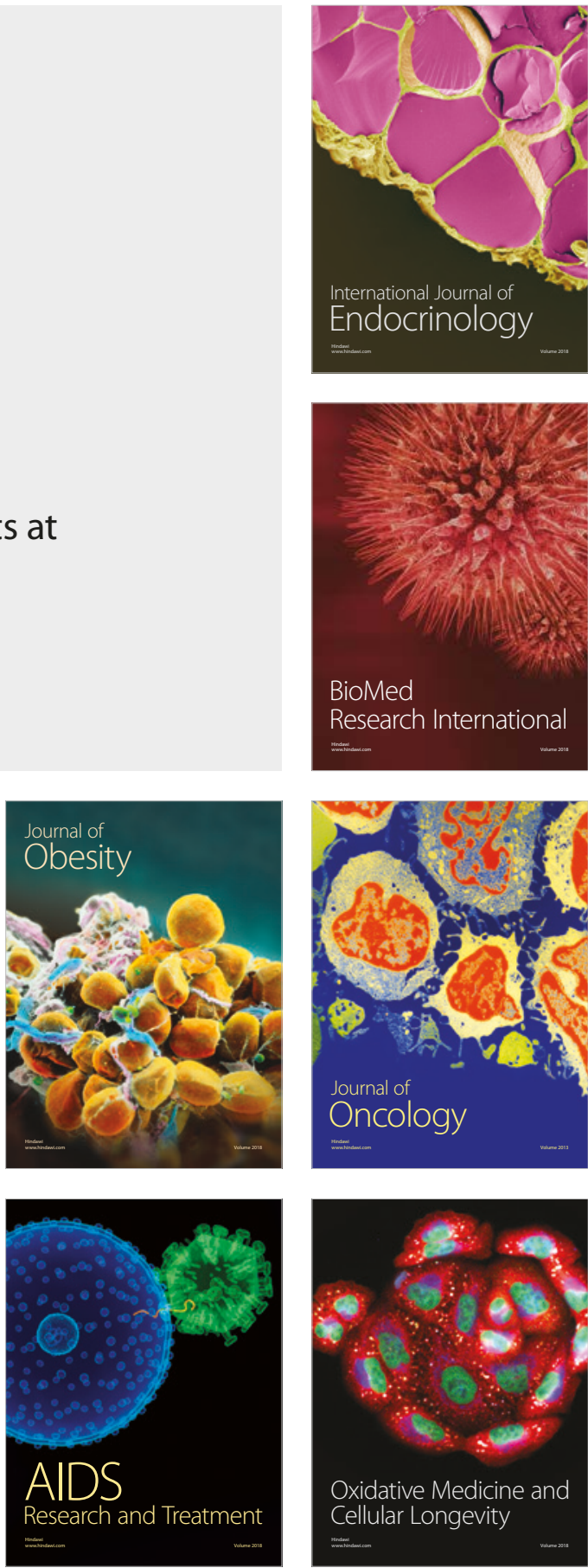